\title{
Neural Basis of Behavior: Pharmacological Effects in Brain Injury and Degenerative Syndromes
}

\author{
Paul R. Sanberg \\ Division of Neurological Surgery, Departments of Surgery, Neurology, Psychiatry, Psychology, \\ Pharmacology and Therapeutics, University of South Florida, College of Medicine, MDC Box 16 \\ 12901 Bruce B. Downs Blvd., Tampa, FL 33612-4799, USA
}

One of the major goals of neuroscience is to develop rational and effective treatments for neurological disorders. Despite technical and conceptual advances in the neurosciences, there are many fundamental questions which are still unanswered. Among the most problematic neurologic disorders are those associated with the loss of brain neurons. Neurodegenerative diseases, congenital disorders, strokes, and penetrating wounds and injury produce unique patterns of behavioral symptoms associated with destruction of specific neuronal populations in the brain and spinal cord. Our understanding of the pathology and molecular biology of these disorders has increased; however, there remains a wide gap between our understanding of the neural substrates of these disorders and our ability to prevent or treat them effectively. With regard to head and spinal cord injuries specifically, the devastating effects can happen so swiftly and with such magnitude that it is important to find treatments which can reduce the nervous system's reaction to the injury and promote full recovery or function.

In the Ninth Annual Symposium on the Neural Basis of Behavior at the annual meeting of the Society for Neuroscience in Anaheim, California, on October 25, 1992, the speakers reviewed and discussed behavioral and functional effects of various putative pharmacological therapies for brain injury and degeneration from basic scientific and clinical perspectives. The purpose of the symposium was to present new evidence and avenues of approach for studying pharmacological strategies for behavioral restoration following brain damage. The symposium was organized by Paul R. Sanberg, University of South Florida and William F. Freed, National Institute of Mental Health, and subsequently chaired and introduced by Paul R. Sanberg. This was followed by an overview of conceptual and practical issues involved in the pharmacological treatment of brain injury by Donald G. Stein, Rutgers University. Dennis M. Feeney, University of New Mexico, described the effects of catecholaminergic drugs on functional recovery from brain injury. Larry Goldstein, Duke University, described similar effects of noradrenergic drugs in brain injury patients. Timothy Schallert, University of Texas at Austin, presented preclinical data in an assymmetrical injury model in rats demonstrating that behavioral events are necessary for neuronal changes to take place. Andrew B. Norman, University of Cincinnati, discussed the role of fetal tissue transplants and glutamate receptor antagonists in influencing recovery in neurodegenerative disorders. Tom J. Walsh, Rutgers University; described antioxidant therapy for producing behavioral recovery. And finally, C. Anthony Altar discussed the effects of BDNF as a therapeutic approach for brain degeneration.

All these investigators have explored a number of avenues which will lead to further understanding for possible treatments of brain injury and neurodegenerative disorders. These included basic scientific and clinical studies which may all prove beneficial in increasing the quality of life in the many patients with neurological disorders. The purpose of the symposium was to review and emphasize these contributions especially as related to behavioral investigations for studying these disorders. The following peer-reviewed articles in this issue also highlight these areas and the potential of these novel psychotherapeutic approaches as treatments for this important public health problem. 

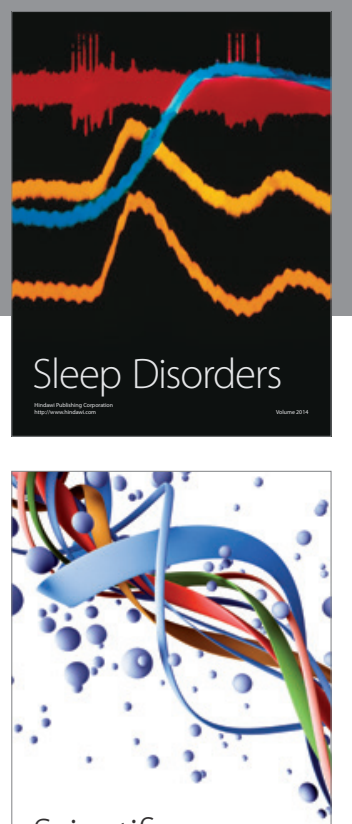

Scientifica
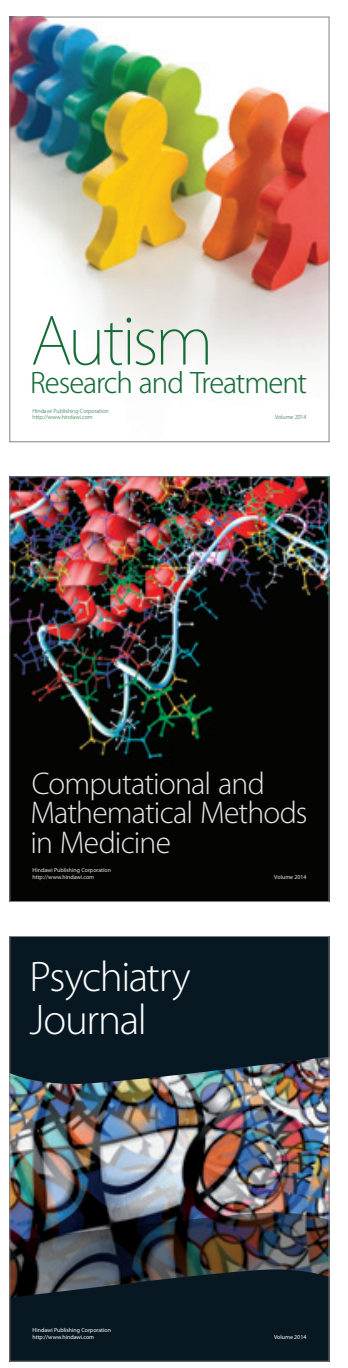
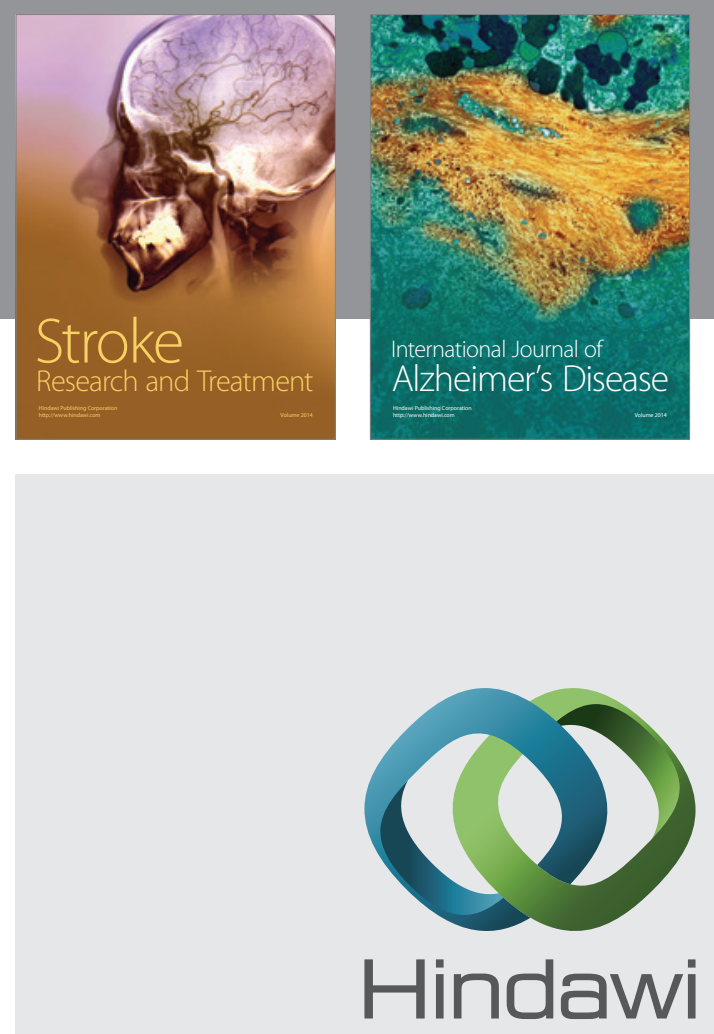

Submit your manuscripts at

http://www.hindawi.com
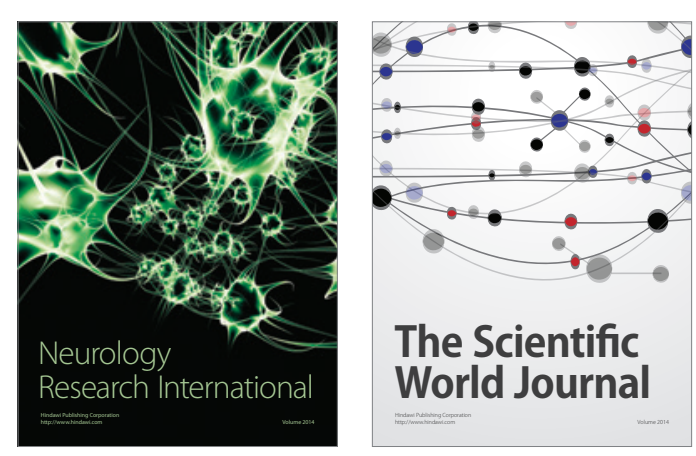

The Scientific World Journal

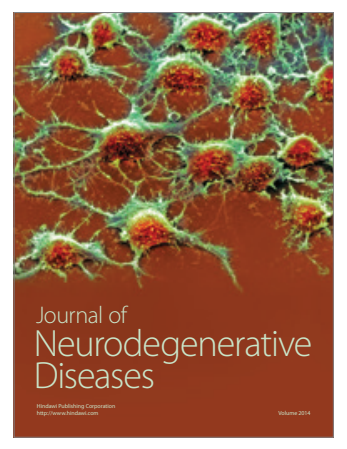

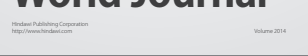

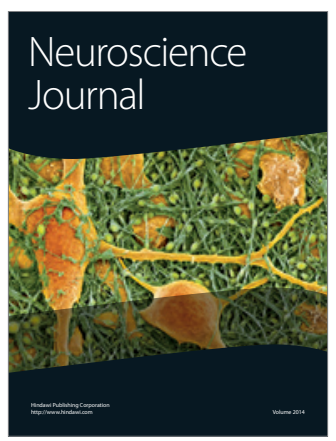

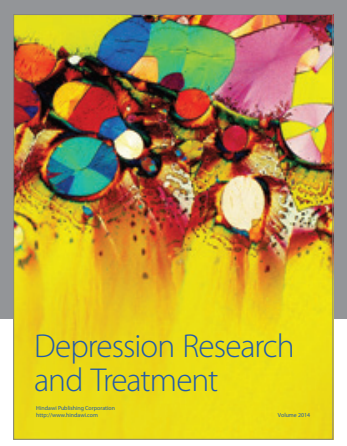
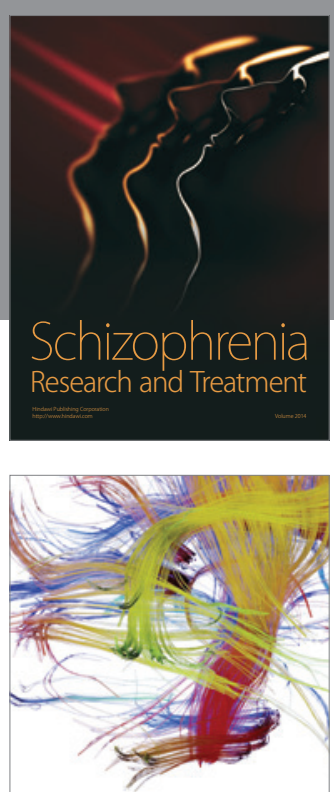

Brain Science

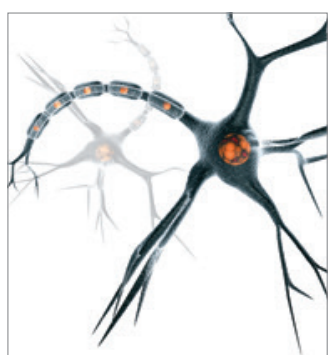

Neural Plasticity
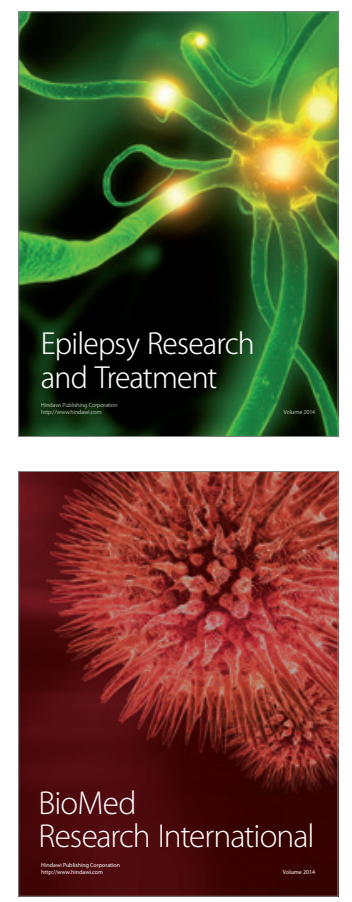

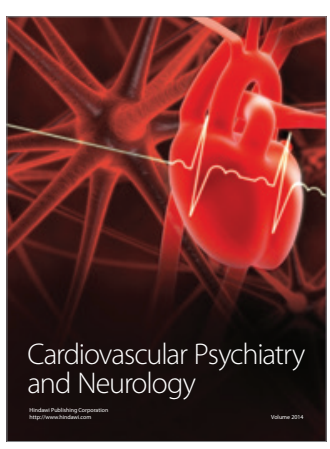

Parkinson's

Disease
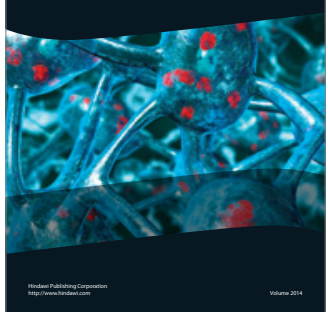